\title{
Magnetic tunneling junctions with an inserted ferromagnetic metal spacer
}

\author{
Sui-Pin Chen*, Ching-Ray Chang \\ Department of Physics, National Taiwan University, Taipei, Taiwan
}

\begin{abstract}
The behavior of the effective spin polarization of the tunneling electrons through an inserted ferromagnetic metal spacer can be regarded as the interference of the spin-up electrons and the spin-down electrons. Instead of using the different insulator, the enhancement of the effective spin polarization can be obtained by merely adjusting the thickness of an inserted ferromagnetic metal spacer. (C) 2002 Elsevier Science B.V. All rights reserved.
\end{abstract}

Keywords: Tunneling magnetoresistance (TMR); Spin polarization

The tunneling magnetoresistance (TMR) is due to spin-polarized electrons tunneling between two ferromagnetic electrodes separated by a thin insulating barrier. Julliere used a simple model for the ferromagnet-insulator-ferromagnet junction, assuming that the tunneling current depends on the density of states of two electrodes, and he concluded that the TMR ratio is proportional to the product of spin polarizations of both ferromagnetic electrodes [1,2]. Slonczewski discussed TMR effects in the light of band theory by analyzing the transmission of charge and spin current through a rectangular barrier of the insulator [3]. He pointed out that the TMR ratio depends not only on spin polarization of both the electrodes, but also on the barrier height. Zhang and Levy studied the ferromagnetinsulator-metal-ferromagnet junction and concluded that the TMR effect will be quenched when the thickness of the inserted metal spacer is beyond a critical thickness [4]. Our work is based on the previous models and is extended to probe the possibility of ferromagnetic inserted metal.

Although, it has been proposed that the TMR ratio may increase with the electrodes of the material with large spin polarization which can be obtained through the spin-filter effect $[5,6]$, until now no experiment has

\footnotetext{
*Corresponding author. Fax: +886-2-3363-9984.

E-mail address: d89222002@ms89.ntu.edu.tw
} (Sui-Pin Chen). been reported. In this article, we propose another magnetic tunneling junction which is the ferromagnetferromagnet-insulator-ferromagnet structure to enhance the TMR ratio by adjusting the thickness of the ferromagnetic spacer, instead of tailoring the barrier height of the insulator.

Two ferromagnetic electrodes separated by a thin ferromagnetic spacer and a tunneling barrier is shown in Fig. 1. The tunneling region Hamiltonian of one spinpolarized conduction electron through the $\mathrm{F}_{1}-\mathrm{F}-\mathrm{I}-\mathrm{F}_{2}$ junction can be modeled as

$H_{\alpha}=-\frac{\hbar^{2}}{2 m^{*}}\left(\frac{\mathrm{d}}{\mathrm{d} \xi}\right)_{\alpha}^{2}+U_{\alpha}-h_{\alpha} \sigma_{\alpha}+\vartheta_{\alpha}$,

where $m^{*}$ is the effective mass, $\hbar$ is the Planck constant, $\xi$ is the tunneling direction and $U$ is the barrier potential of the insulator. Subscript $\alpha=1(2, i, b)$ represents the right ferromagnetic layer $F_{1}$ (the left ferromagnetic layer $\mathrm{F}_{2}$, the ferromagnetic spacer $\mathrm{F}$, and the insulator barrier I). The internal exchange energy of the $\alpha$ th ferromagnetic layer is $-h_{\alpha} \sigma_{\alpha}$ with $-h$ denoting the molecular field and $\sigma$ being the Pauli spin operator. The term $\vartheta_{\alpha}$ is the potential due to the contact between two differential materials and let $\vartheta_{1}$ be the zero point. In practice, $m^{*}$ may differ within four regions; for simplicity, we assume that the effective mass is the same for all materials. The directions $h_{1}$ and $h_{2}$ are along the corresponding spin quantization axes $z$ and $z^{\prime}$ with a deviation $\phi$, and the $z$ axis remains fixed in our calculations [3]. We use the 


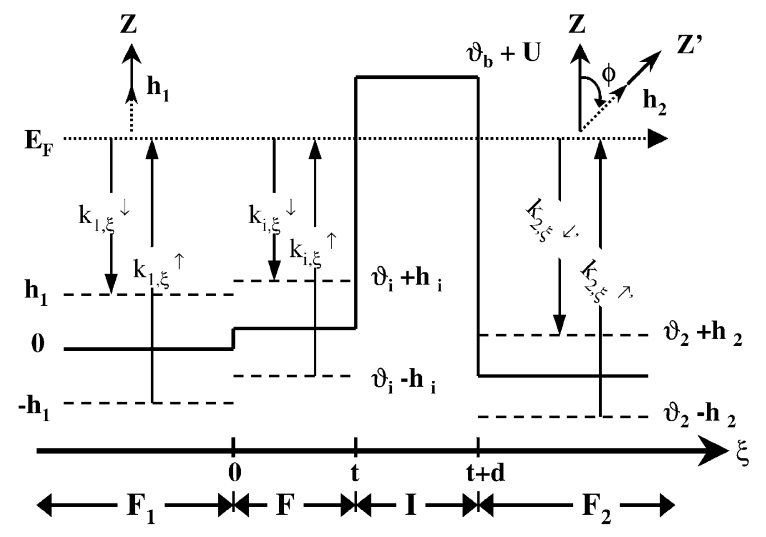

Fig. 1. Schematic diagram for an $\mathrm{F}_{1}-\mathrm{F}-\mathrm{I}-\mathrm{F}_{2}$ tunneling junction.

same spin quantization axis for the $\mathrm{F}_{1}$-layer and the $\mathrm{F}$ layer, because the ferromagnetic spacer is assumed to be pinned by the left ferromagnetic electrode.

Considering the spinor transformation between all the interfaces, the transmission probability $T_{k^{\sigma} \rightarrow k_{2}^{\sigma^{\prime}}}$ of the conduction electron across the spin-filter tunneling junction can be determined from an incident plane wave of the spin-up electron in the $F_{1}$ layer by matching the boundary conditions imposed on the wave function and its first derivate at the interfaces, where $\sigma$ and $\sigma^{\prime}$ are based on the $z$ - and $z^{\prime}$-axis, respectively $[3,4]$.

$T_{k_{1, \xi}^{\uparrow} \rightarrow k_{2, \xi}^{\uparrow}}(\phi)=8 \frac{k_{1, \xi}^{\uparrow} k_{2, \xi}^{\uparrow} \kappa^{2} \mathrm{e}^{-2 \kappa d}}{A\left[\left(k_{2, \xi}^{\uparrow}\right)^{2}+(\kappa)^{2}\right]}(1+\cos \phi)$,

$T_{k_{1, \xi}^{\uparrow} \rightarrow k_{2, \xi}^{\downarrow}}(\phi)=8 \frac{k_{1, \xi}^{\uparrow} k_{2, \xi}^{\downarrow} \kappa^{2} \mathrm{e}^{-2 \kappa d}}{A\left[\left(k_{2, \xi}^{\downarrow}\right)^{2}+(\kappa)^{2}\right]}(1-\cos \phi)$,

where

$$
\begin{aligned}
A= & {\left[\left(k_{1, \xi}^{\uparrow}\right)^{2}+(\kappa)^{2}\right] \cos ^{2}\left(k_{i, \xi}^{\uparrow} t\right) } \\
& +\left[\left(k_{i, \xi}^{\uparrow}\right)^{2}+\frac{\left(k_{1, \xi}^{\uparrow}\right)^{2}(\kappa)^{2}}{\left(k_{i, \xi}^{\uparrow}\right)^{2}}\right] \sin ^{2}\left(k_{i, \xi}^{\uparrow} t\right) \\
& +\left[k_{i, \xi}^{\uparrow} \kappa-\frac{\left(k_{1, \xi}^{\uparrow}\right)^{2} \kappa}{k_{i, \xi}^{\uparrow}}\right] \sin \left(2 k_{i, \xi}^{\uparrow} t\right), \\
\left(k_{\alpha}^{\sigma}\right)^{2}= & \left(k_{\alpha, \xi}^{\sigma}\right)^{2}+\left(k_{\alpha, \rho}^{\sigma}\right)^{2} \\
= & \frac{2 m^{*}}{\hbar^{2}}\left(H_{\alpha}+h_{\alpha} \sigma_{\alpha}-\vartheta_{\alpha}\right), \\
\kappa= & \sqrt{\frac{2 m^{*}}{\hbar^{2}}\left(U_{\mathrm{b}}+\vartheta_{\mathrm{b}}-H_{\mathrm{b}}\right) .}
\end{aligned}
$$

The length $d$ and $t$ refer to the thickness of the insulator and that of the ferromagnetic spacer, respectively. Here, $k_{\alpha}^{\sigma}$ is the wave vector of the conduction electron from the $\mathrm{F}_{1}$ layer with the transverse component $k_{\alpha, \rho}^{\sigma}$ and the longitudinal component $k_{\alpha, \xi}^{\sigma}$. The conservation of momentum parallel to the interface is required in our calculation. From the consideration of the symmetry, we can easily obtain $T_{k_{1, \xi}^{\downarrow} \rightarrow k_{2, \xi}^{\downarrow}}(\phi)$ and $T_{k_{1, \xi}^{\downarrow} \rightarrow k_{2, \xi}^{\dagger}}(\phi)$. Since electrons with $k_{\alpha, \xi}^{\sigma}$ near the Fermi level $\left(E_{\mathrm{F}}\right)$ may carry most of the current at the zero temperature and the vanishing bias, we regard the energy of the conduction electron as $E_{\mathrm{F}}$ in calculating the tunneling conductance $[3,7]$, while the total transmission probability and the corresponding conductance are

$$
\begin{aligned}
T(\phi) & =\sum_{\sigma, \sigma^{\prime}} T_{k_{1, \xi}^{\sigma} \rightarrow k_{2, \xi}^{\sigma^{\prime}}} \\
& =T_{p}(1+\varepsilon \cos \phi)
\end{aligned}
$$

and

$G(\phi)=\frac{e^{2} \kappa}{8 \pi^{2} \hbar d} T(\phi)$.

$T_{\mathrm{p}}$ is the mean transmission probability, $e$ is the electron charge and

$$
\begin{aligned}
\varepsilon= & P_{\mathrm{F}_{1} \mathrm{FI}} P_{\mathrm{F}_{2} \mathrm{I}}, \\
P_{\mathrm{F}_{1} \mathrm{FI}} & =\frac{k_{1}^{\uparrow} B-k_{1}^{\downarrow} C}{k_{1}^{\uparrow} B+k_{1}^{\downarrow} C}, \\
P_{\mathrm{F}_{2} \mathrm{FI}} & =\frac{k_{2}^{\uparrow}-k_{2}^{\downarrow}}{k_{2}^{\uparrow}+k_{2}^{\downarrow}} \frac{\kappa^{2}-k_{2}^{\uparrow} k_{2}^{\downarrow}}{\kappa^{2}+k_{2}^{\uparrow} k_{2}^{\downarrow},} \\
B= & {\left[\left(k_{1}^{\downarrow}\right)^{2}+(\kappa)^{2}\right] \cos ^{2}\left(k_{i}^{\downarrow} t\right) } \\
+ & {\left[\left(k_{i}^{\downarrow}\right)^{2}+\frac{\left(k_{1}^{\downarrow}\right)^{2}(\kappa)^{2}}{\left(k_{i}^{\downarrow}\right)^{2}}\right] \sin ^{2}\left(k_{i}^{\downarrow} t\right) } \\
+ & {\left[k_{i}^{\downarrow} \kappa-\frac{\left(k_{1}^{\downarrow}\right)^{2} \kappa}{k_{i}^{\downarrow}}\right] \sin \left(2 k_{i}^{\downarrow} t\right), } \\
C= & {\left[\left(k_{1}^{\uparrow}\right)^{2}+(\kappa)^{2}\right] \cos ^{2}\left(k_{i}^{\uparrow} t\right) } \\
+ & {\left[\left(k_{i}^{\uparrow}\right)^{2}+\frac{\left(k_{1}^{\uparrow}\right)^{2}(\kappa)^{2}}{\left(k_{i}^{\uparrow}\right)^{2}}\right] \sin ^{2}\left(k_{i}^{\uparrow} t\right) } \\
& +\left[k_{i}^{\uparrow} \kappa-\frac{\left(k_{1}^{\uparrow}\right)^{2} \kappa}{k_{i}^{\uparrow}}\right] \sin \left(2 k_{i}^{\uparrow} t\right) .
\end{aligned}
$$

For the detailed analysis of Eq. (11) [8], we found that with $k_{i}^{\uparrow} \cong k_{i}^{\downarrow}$

$$
\begin{aligned}
& P_{\mathrm{F}_{1} \mathrm{FI}} \cong \frac{1}{2} \frac{k_{1}^{\uparrow}-k_{1}^{\downarrow}}{k_{1}^{\uparrow}+k_{1}^{\downarrow}} \sin \left(2 k_{i}^{\uparrow} t+\theta_{\uparrow}+\pi\right) \\
&+\frac{1}{2} \frac{k_{1}^{\uparrow}-k_{1}^{\downarrow}}{k_{1}^{\uparrow}+k_{1}^{\downarrow}} \sin \left(2 k_{i}^{\downarrow} t+\theta_{\downarrow}+\pi\right), \\
& \theta_{\uparrow}=\sin ^{-1} \frac{\left(k_{i}^{\uparrow}\right)^{4}-(\kappa)^{2} k_{1}^{\uparrow} k_{1}^{\downarrow}}{\left(k_{i}^{\uparrow}\right)^{4}+(\kappa)^{2} k_{1}^{\uparrow} k_{1}^{\downarrow}}, \\
& \theta_{\downarrow}=\sin ^{-1} \frac{\left(k_{i}^{\downarrow}\right)^{4}-(\kappa)^{2} k_{1}^{\uparrow} k_{1}^{\downarrow}}{\left(k_{i}^{\downarrow}\right)^{4}+(\kappa)^{2} k_{1}^{\uparrow} k_{1}^{\downarrow}} .
\end{aligned}
$$




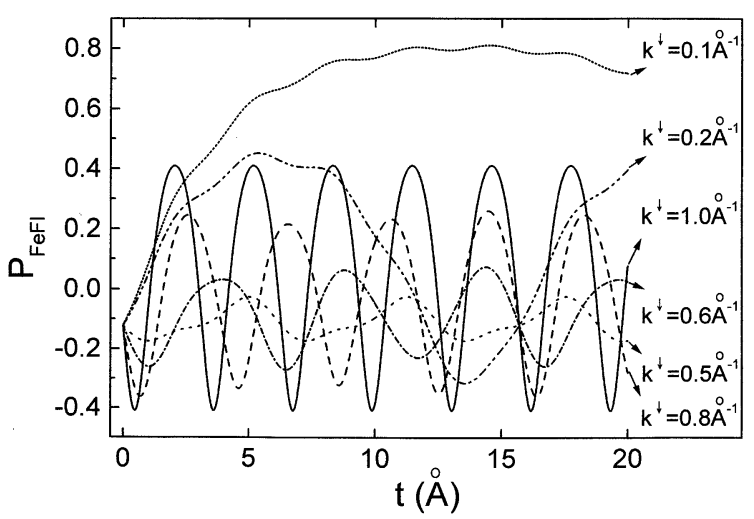

Fig. 2. The effective spin polarization of the $\mathrm{Fe}-\mathrm{F}-\mathrm{I}$ region in an $\mathrm{Fe}-\mathrm{F}-\mathrm{I}-\mathrm{F}_{2}$ junction versus the thickness $t$ of the ferromagnetic spacer.

Since the energy of the tunneling conduction electrons from electrodes is near the Fermi level, the above wave vectors reduce to the Fermi wave vectors in different regions. Then, the tunneling magnetic resistance (TMR) ratio of the $\mathrm{F}_{1}-\mathrm{F}-\mathrm{I}-\mathrm{F}_{2}$ junction is

$\frac{\Delta R}{\bar{R}}=2 \varepsilon$,

where $\Delta R$ is the difference magnetic resistance between the parallel and antiparallel magnetizations of two ferromagnetic electrodes, and $\bar{R}$ is the mean magnetic resistance.

Fig. 2 shows the effective spin polarization of conduction electrons through the $\mathrm{Fe}-\mathrm{F}-\mathrm{I}$ region of the $\mathrm{Fe}-\mathrm{F}-\mathrm{I}-\mathrm{F}_{2}$ junction, $P_{\mathrm{FeFI}}$, and it depends on the thickness of the ferromagnetic spacer. These calculations are arbitrarily taken as the $\mathrm{Fe}-\mathrm{F}\left(k_{i}^{\uparrow}=1 \AA^{-1}\right)-\mathrm{I}(\kappa=$ $\left.0.5 \AA^{-1}\right)$ region with various $k_{i}^{\downarrow}$ of the F-layer, where the Fermi wave vectors of $\mathrm{Fe}$ for spin-up and spin-down are 1.50 and $0.44 \AA^{-1}$, respectively $[9,10]$. As $k_{i}^{\downarrow}$ of the F-layer decrease from 1 to $0.5 \AA^{-1}$, the peak value of $P_{\text {FeFI }}$ also decreases; however, as $k_{i}^{\downarrow}$ decreases from 0.5 to $0.1 \AA^{-1}$, the peak value of $P_{\mathrm{FeFI}}$ increases.

From Eq. (15), we can roughly attribute the behavior of the effective spin polarization of the $\mathrm{F}_{1}-\mathrm{F}-\mathrm{I}$ interface to the superpositions of the amplitude oscillation of the spin polarization of the $F_{1}$ layer with two wavelengths of the $\mathrm{F}$ spacer, $\pi / k_{i}^{\uparrow}$ and $\pi / k_{i}^{\downarrow}$, and their corresponding initial phases $\theta^{\uparrow}$ and $\theta_{\downarrow}$, respectively. Therefore, in Fig. 2, the small period is from $k_{i}^{\uparrow}$ and the large period is from $k_{i}^{\downarrow}$. On the other hand, the influence of the barrier height is on the phase of the oscillation of the effective spin polarization from what we observe of Eqs. (16) and (17).

The effective spin polarization of the $\mathrm{F}_{1}-\mathrm{I}$ interface of the conventional $\mathrm{F}_{1}-\mathrm{I}-\mathrm{F}_{2}$ junction is the product of the spin polarization of the $\mathrm{F}_{1}$-layer and an interfacial factor. The magnitude of interfacial factor is always less than 1 due to the coupling between the ferromagnet and the insulator and it is very sensitive to the barrier height in the Slonczewski's model [3]. Therefore, it is difficult to obtain the maximum effective spin polarization by varying the barrier height. An extra inserted ferromagnetic layer in the Fe-F-I interface is quiet useful, since it is easy to obtain a large effective spin polarization by merely adjusting the thickness of the inserted ferromagnetic spacer. Sometimes, with an appropriate inserted layer thickness in the Fe-F-I interface, the effective polarization can be larger than $41 \%$, which is the upper limit in the Fe-I interface from Slonczewski's model.

\section{References}

[1] M. Julliere, Phys. Lett. 54A (1975) 225.

[2] R.M. Tedrow, R. Meservey, Phys. Rev. Lett. 26 (1971) 192.

[3] J.C. Slonczewski, Phys. Rev. B 39 (1989) 6995.

[4] S. Zhang, P.M. Levy, Phys. Rev. Lett. 25 (1998) 5660.

[5] X. Hao, J.S. Moodera, R. Meservey, Phys. Rev. B. 42 (1990) 8235.

[6] Ching-Ray Chang, Sui-Pin Chen, J. Magn. Magn. Mater. 209 (2000) 61.

[7] C.B. Duck, Tunneling in Solid, Academic Press, New York, 1969.

[8] Ching-Ray Chang, Sui-Pin Chen, unpublished.

[9] H.P.J. Wijn (Ed.), Magnetic Properties of Metals: dElement, Alloys, and Compounds, Springer, Heidelberg, 1991, p. 17.

[10] D. Grunder, Phys. Rev. Lett. 86 (2001) 1058. 